TAIWANESE JOURNAL OF MATHEMATICS

Vol. 11, No. 1, pp. 231-237, March 2007

This paper is available online at http://www.math.nthu.edu.tw/tjm/

\title{
LOGARITHMIC CONVEXITY OF THE ONE-PARAMETER MEAN VALUES
}

\author{
Wing-Sum Cheung* and Feng Qi**
}

\begin{abstract}
In this article, the logarithmic convexity of the one-parameter mean values $J(r)$ and the monotonicity of the product $J(r) J(-r)$ with $r \in \mathbb{R}$ are presented. Some more general results are established.
\end{abstract}

\section{INTRODUCTION}

The one-parameter mean values $J(r ; x, y)$ for two positive numbers $x$ and $y$ with $x \neq y$ are defined by

$$
J(r) \triangleq J(r ; x, y)= \begin{cases}r\left(x^{r+1}-y^{r+1}\right) /(r+1)\left(x^{r}-y^{r}\right), & r \neq 0,-1 \\ (x-y) /(\ln x-\ln y), & r=0 ; \\ x y(\ln x-\ln y) /(x-y), & r=-1 .\end{cases}
$$

There has been some literature on the one-parameter mean values $J(r ; x, y)$, see $[1-4,7]$.

The main purpose of this paper is to prove the logarithmic convexity of the oneparameter mean values $J(r ; x, y)$ and the monotonicity of $J(-r) J(r)$ for $r \in \mathbb{R}$.

Our main results are as follows.

Theorem 1. Let $x$ and $y$ be positive numbers with $x \neq y$. Then

(i) The one-parameter mean values $J(r)$ are strictly increasing in $r \in \mathbb{R}$;

Received January 24, 2005, accepted January 23, 2006.

Communicated by H. M. Srivastava.

2000 Mathematics Subject Classification: Primary 26A48, 26A51; Secondary 26B25, 26 D07.

Key words and phrases: Logarithmic convexity, Monotonicity, One-parameter mean values.

*Supported by a seed grant for basic research of the University of Hong Kong, Hong Kong SAR, People's Republic of China.

**Supported by the Science Foundation of Project for Fostering Innovation Talents at Universities of Henan Province of the People's Republic of China 
(ii) The one-parameter mean values $J(r)$ are strictly logarithmically convex in $(-\infty,-1 / 2)$ and strictly logarithmically concave in $(-1 / 2, \infty)$.

Theorem 2. Let $\mathcal{J}(r)=J(r) J(-r)$ with $r \in \mathbb{R}$ for fixed positive numbers $x$ and $y$ with $x \neq y$. Then the function $\mathcal{J}(r)$ is strictly increasing in $(-\infty, 0)$ and strictly decreasing in $(0, \infty)$.

\section{Proofs of Theorems}

\subsection{Proof of Theorem 1 .}

Let

$$
g(t) \triangleq g(t ; x, y)= \begin{cases}\left(y^{t}-x^{t}\right) / t, & t \neq 0 \\ \ln y-\ln x, & t=0\end{cases}
$$

for positive numbers $x$ and $y$ with $x \neq y$.

In [5], Corollary 3 states that, for $y>x>0$, if $t>0$, then

$$
g^{2}(t) g^{\prime \prime \prime}(t)-3 g(t) g^{\prime}(t) g^{\prime \prime}(t)+2\left[g^{\prime}(t)\right]^{3}<0 ;
$$

if $t<0$, inequality (3) reverses.

2.1.1. Formula (3) implies that, for $y>x>0$,

$$
\left[g^{\prime}(t) / g(t)\right]^{\prime \prime}=\operatorname{sgn}(-t) .
$$

From this, we obtain that the function $\left[g^{\prime}(t) / g(t)\right]^{\prime}$ is strictly increasing in $(-\infty, 0)$ and strictly decreasing in $(0, \infty)$.

By using Cauchy-Schwartz integral inequality or Tchebycheff integral inequality, it is obtained [6-8] that $\left[g^{\prime}(t) / g(t)\right]^{\prime}>0$ for $t \in \mathbb{R}$. Then the function $g^{\prime}(t) / g(t)$ is strictly increasing in $(-\infty, \infty)$.

The one-parameter mean values $J(r)$ can be rewritten in terms of $g$ as $J(r)=$ $g(r+1) / g(r)$ with $r \in \mathbb{R}$ for $y>x>0$. Taking the logarithm of $J(r)$ yields

(5) $\quad \ln J(r)=\ln g(r+1)-\ln g(r)=\int_{r}^{r+1} \frac{g^{\prime}(u)}{g(u)} \mathrm{d} u=\int_{0}^{1} \frac{g^{\prime}(u+r)}{g(u+r)} \mathrm{d} u$

and $[\ln J(r)]^{\prime}=g^{\prime}(r+1) / g(r+1)-g^{\prime}(r) / g(r)>0$. Hence the functions $\ln J(r)$ and $J(r)$ are strictly increasing in $r \in(-\infty, \infty)$. This proves (i). 
2.1.2. If $r<-1$, then $r<r+1<0$ and $[\ln J(r)]^{\prime \prime}=\left[g^{\prime}(r+1) / g(r+1)\right]^{\prime}-$ $\left[g^{\prime}(r) / g(r)\right]^{\prime}>0$ which follows from the strictly increasing property of $\left[g^{\prime}(r) / g(r)\right]^{\prime}$ in $(-\infty, 0)$.

If $r>0$, then from the strictly decreasing property of $[g(r) / g(r)]^{\prime}$ in $(0, \infty)$, we have $[\ln J(r)]^{\prime \prime}<0$.

If $-1<r<0$, then $r<0<r+1$, and we have

$$
\begin{aligned}
{[\ln J(r)]^{\prime \prime} } & =\left(\frac{g^{\prime}(r+1)}{g(r+1)}\right)^{\prime}-\left(\frac{g^{\prime}(r)}{g(r)}\right)^{\prime} \\
& =\frac{g^{\prime \prime}(r+1) g(r+1)-\left[g^{\prime}(r+1)\right]^{2}}{g^{2}(r+1)}-\frac{g^{\prime \prime}(r) g(r)-\left[g^{\prime}(r)\right]^{2}}{g^{2}(r)} \\
& =\frac{g^{\prime \prime}(u) g(u)-\left[g^{\prime}(u)\right]^{2}}{g^{2}(u)}-\frac{g^{\prime \prime}(-r) g(-r)-\left[g^{\prime}(-r)\right]^{2}}{g^{2}(-r)} \\
& =\frac{g^{\prime \prime}(u) g(u)-\left[g^{\prime}(u)\right]^{2}}{g^{2}(u)}-\frac{g^{\prime \prime}(v) g(v)-\left[g^{\prime}(v)\right]^{2}}{g^{2}(v)} \\
& =\left(\frac{g^{\prime}(u)}{g(u)}\right)^{\prime}-\left(\frac{g^{\prime}(v)}{g(v)}\right)^{\prime}
\end{aligned}
$$

where $u=r+1>0$ and $v=-r>0$. Thus, $[\ln J(r)]^{\prime \prime}<0$ for $-1<r<0$ and $r+1>-r$. This means that $[\ln J(r)]^{\prime \prime}<0$ for $r \in(-1 / 2,0)$.

Similar as above, $[\ln J(r)]^{\prime \prime}>0$ for $-1<r<0$ and $-r>r+1$. This means that $[\ln J(r)]^{\prime \prime}>0$ for $r \in(-1,-1 / 2)$. This proves (ii).

Remark. From (4), (5) and by direct calculation, we have

$$
[\ln J(r)]^{\prime \prime}=\int_{0}^{1} \frac{\mathrm{d}^{2}}{\mathrm{~d} r^{2}}\left(\frac{g^{\prime}(u+r)}{g(u+r)}\right) \mathrm{d} u<0
$$

for $r \in(0, \infty)$. This means that $J(r ; x, y)$ is strictly logarithmically concave in $r \in(0, \infty)$, whether $x>y$ or $x<y$, since $J(r ; x, y)=J(r ; y, x)$ holds.

By straightforward computation, we have

$$
J(r)=\frac{x y}{J(-r-1)}
$$

for $r \in \mathbb{R}$. Hence, if $r \in(-\infty,-1)$, from (3), (4) and (7), it follows that $[\ln J(r)]^{\prime \prime}=-[\ln J(-r-1)]^{\prime \prime}=-\int_{0}^{1}\left\{\mathrm{~d}^{2}\left[g^{\prime}(u-r-1) / g(u-r-1)\right] / \mathrm{d} r^{2}\right\} \mathrm{d} u>$ 0 . This tells us that the one-parameter mean values $J(r ; x, y)$ are strictly logarithmically convex in $r \in(-\infty,-1)$, whether $x>y$ or $x<y$, since $J(r ; x, y)=$ $J(r ; y, x)$. 


\subsection{Proof of Theorem 2 .}

It is easy to obtain that $\mathcal{J}(r)=x y J(r) / J(r-1)$ for $r \in \mathbb{R}$. Then $\ln \mathcal{J}(r)=$ $\ln (x y)+\ln J(r)-\ln J(r-1)$ and

$$
[\ln \mathcal{J}(r)]^{\prime}=\frac{J^{\prime}(r)}{J(r)}-\frac{J^{\prime}(r-1)}{J(r-1)} .
$$

Theorem 1 states that the function $J(r)$ is strictly logarithmically convex in $(-\infty,-1 / 2)$. Thus, being the derivative of $\ln J(r), J^{\prime}(r) / J(r)$ is strictly increasing in $(-\infty,-1 / 2)$, that is, $J^{\prime}(r) / J(r)>J^{\prime}(r-1) / J(r-1)$, or, equivalently, $[\ln \mathcal{J}(r)]^{\prime}>0$ for $r \in(-\infty,-1 / 2)$, thus $\ln \mathcal{J}(r)$ and $\mathcal{J}(r)$ are strictly increasing in $(-\infty,-1 / 2)$.

From (8), it follows that $\ln J(r)=\ln (x y)-\ln J(-r-1)$ and $J^{\prime}(r) / J(r)=$ $J^{\prime}(-r-1) / J(-r-1)$. Then (9) results in $[\ln \mathcal{J}(r)]^{\prime}=J^{\prime}(-r-1) / J(-r-1)-$ $J^{\prime}(r-1) / J(r-1)$.

For $r \in(-1 / 2,0)$, we have $-3 / 2<r-1<-1$ and $-1<-r-1<-1 / 2$. Since $J^{\prime}(r) / J(r)$ is strictly increasing in $(-\infty,-1 / 2)$, $[\ln \mathcal{J}(r)]^{\prime}>0$ for $r \in$ $(-1 / 2,0)$, therefore $\ln \mathcal{J}(r)$ and $\mathcal{J}(r)$ are also strictly increasing in $(-1 / 2,0)$.

It is clear that the function $\mathcal{J}(r)$ is even in $(-\infty, \infty)$. So, it is easy to see that $\mathcal{J}(r)$ is strictly decreasing in $(0, \infty)$. The proof of Theorem 2 is completed.

\section{Some Related Results}

For $x \neq y$ and $\alpha>0$, define for $r \in \mathbb{R}$

$$
J_{\alpha}(r) \triangleq J_{\alpha}(r ; x, y)= \begin{cases}{\left[r\left(x^{r+\alpha}-y^{r+\alpha}\right) /(r+\alpha)\left(x^{r}-y^{r}\right)\right]^{1 / \alpha},} & r \neq 0,-\alpha \\ {\left[\left(x^{\alpha}-y^{\alpha}\right) / \alpha(\ln x-\ln y)\right]^{1 / \alpha},} & r=0 ; \\ {\left[\alpha x^{\alpha} y^{\alpha}(\ln x-\ln y) /\left(x^{\alpha}-y^{\alpha}\right)\right]^{1 / \alpha},} & r=-\alpha .\end{cases}
$$

We call $J_{\alpha}(r ; x, y)$ the generalized one-parameter mean values for two positive numbers $x$ and $y$ in the interval $(-\infty, \infty)$.

It is clear that $J_{1}(r ; x, y)=J(r ; x, y)$ and $J_{\alpha}(r ; x, y)=[g(r+\alpha) / g(r)]^{1 / \alpha}$. By the same arguments as in the proofs of Theorem 1 and Theorem 2, we can obtain the following

Theorem 3. Let $x$ and $y$ be positive numbers with $x \neq y$. Then

(1) The generalized one-parameter mean values $J_{\alpha}(r)$ are strictly increasing in $r \in \mathbb{R}$

(2) The mean values $J_{\alpha}(r)$ are strictly logarithmically convex in $(-\infty,-\alpha / 2)$ and strictly logarithmically concave in $(-\alpha / 2, \infty)$; 
(3) Let $\mathcal{J}_{\alpha}(r)=J_{\alpha}(r) J_{\alpha}(-r)$ with $r \in \mathbb{R}$ for positive numbers $x$ and $y$ with $x \neq y$. Then the function $\mathcal{J}_{\alpha}(r)$ is strictly increasing in $(-\infty, 0)$ and strictly decreasing in $(0, \infty)$.

Proof. These follow from combining the identities $\left[J_{\alpha}(r ; x, y)\right]^{\alpha}=J\left(r / \alpha ; x^{\alpha}\right.$, $\left.y^{\alpha}\right)$ and $\left[\mathcal{J}_{\alpha}(r)\right]^{\alpha}=\mathcal{J}(r / \alpha)$ with Theorem 1 and Theorem 2.

Theorem 4. The function $(r+\alpha)\left[J_{\alpha}(r)\right]^{\alpha}$ is strictly increasing and strictly convex in $(-\infty, \infty)$, and is strictly logarithmically concave for $r>-\alpha / 2$.

Proof. Direct computation gives

$$
\begin{aligned}
(r+\alpha)\left[J_{\alpha}(r ; x, y)\right]^{\alpha} & =\alpha\left(\frac{r}{\alpha}+1\right) J\left(\frac{r}{\alpha} ; x^{\alpha}, y^{\alpha}\right), \\
\frac{\mathrm{d}^{2} \ln \left\{(r+\alpha)\left[J_{\alpha}(r)\right]^{\alpha}\right\}}{\mathrm{d} r^{2}} & =-\frac{1}{(r+\alpha)^{2}}+\alpha\left[\ln J_{\alpha}(r)\right]^{\prime \prime} .
\end{aligned}
$$

From the result by Alzer in [3] that the function $(r+1) J(r ; x, y)$ is strictly convex in $(-\infty, \infty)$, it is not difficult to obtain that the function $(r+\alpha)\left[J_{\alpha}(r ; x, y)\right]^{\alpha}$ is also strictly convex in $(-\infty, \infty)$ by using (11).

By standard argument, we have

$$
\begin{aligned}
\lim _{r \rightarrow-\infty}\left\{\left[J_{\alpha}(r)\right]^{\alpha}\right\}^{\prime}= & \lim _{r \rightarrow-\infty}\left[\alpha\left(z^{r+\alpha}-1\right) /(r+\alpha)\left(z^{r}-1\right)\right] \\
& -\lim _{r \rightarrow-\infty}\left[r z^{r}\left(z^{\alpha}-1\right) \ln z /\left(z^{r}-1\right)^{2}\right]=0
\end{aligned}
$$

and $\lim _{r \rightarrow-\infty}\left[J_{\alpha}(r)\right]^{\alpha}=\min \left\{x^{\alpha}, y^{\alpha}\right\}$, where $z=y / x \neq 1$. This leads to

$$
\begin{aligned}
\lim _{r \rightarrow-\infty}\left\{(r+\alpha)\left[J_{\alpha}(r)\right]^{\alpha}\right\}^{\prime} & =\lim _{r \rightarrow-\infty}\left[J_{\alpha}(r)\right]^{\alpha}+\lim _{r \rightarrow-\infty}(r+\alpha)\left\{\left[J_{\alpha}(r)\right]^{\alpha}\right\}^{\prime} \\
& =\min \left\{x^{\alpha}, y^{\alpha}\right\}>0 .
\end{aligned}
$$

The convexity of $(r+\alpha)\left[J_{\alpha}(r)\right]^{\alpha}$ means that $\left\{(r+\alpha)\left[J_{\alpha}(r)\right]^{\alpha}\right\}^{\prime}$ is strictly increasing, in view of (13), $\left\{(r+\alpha)\left[J_{\alpha}(r)\right]^{\alpha}\right\}^{\prime}>0$, and so $(r+\alpha)\left[J_{\alpha}(r)\right]^{\alpha}$ is strictly increasing in $(-\infty, \infty)$.

Since $J_{\alpha}(r)$ is strictly logarithmically concave in $(-\alpha / 2, \infty),\left[\ln J_{\alpha}(r)\right]^{\prime \prime}<$ 0 , then $\mathrm{d}^{2} \ln \left\{(r+\alpha)\left[J_{\alpha}(r)\right]^{\alpha}\right\} / \mathrm{d} r^{2}<0$ by (12). This means that the function $(r+\alpha)\left[J_{\alpha}(r)\right]^{\alpha}$ is strictly logarithmically concave in $(-\alpha / 2, \infty)$.

Corollary 1. If $r<-\alpha$, then

$$
0<\frac{\left\{\left[J_{\alpha}(r)\right]^{\alpha}\right\}^{\prime}}{\left[J_{\alpha}(r)\right]^{\alpha}}=\frac{\left\{\left[J_{\alpha}(-r-\alpha)\right]^{\alpha}\right\}^{\prime}}{\left[J_{\alpha}(-r-\alpha)\right]^{\alpha}}<-\frac{1}{r+\alpha},
$$




$$
0<\frac{\left\{\left[J_{\alpha}(r)\right]^{\alpha}\right\}^{\prime \prime}}{\left\{\left[J_{\alpha}(r)\right]^{\alpha}\right\}^{\prime}}<-\frac{2}{r+\alpha} .
$$

Proof. From the monotonicity and convexity of $(r+\alpha) J_{\alpha}(r)$, we have

$$
\begin{gathered}
\left\{(r+\alpha)\left[J_{\alpha}(r)\right]^{\alpha}\right\}^{\prime}=\left[J_{\alpha}(r)\right]^{\alpha}+(r+\alpha)\left\{\left[J_{\alpha}(r)\right]^{\alpha}\right\}^{\prime}>0, \\
\left\{(r+\alpha)\left[J_{\alpha}(r)\right]^{\alpha}\right\}^{\prime \prime}=2\left\{\left[J_{\alpha}(r)\right]^{\alpha}\right\}^{\prime}+(r+\alpha)\left\{\left[J_{\alpha}(r)\right]^{\alpha}\right\}^{\prime \prime}>0 .
\end{gathered}
$$

Inequality (14) follows from combining (16) with $\left[J_{\alpha}(r)\right]^{\alpha}=x y /\left[J_{\alpha}(-r-\alpha)\right]^{\alpha}$. Inequality (15) is a direct consequence of (17).

Theorem 5. The function $r \ln J_{\alpha}(r)$ is strictly convex in $(-\alpha / 2,0)$.

Proof. Direct calculation yields $\left[r \ln J_{\alpha}(r)\right]^{\prime \prime}=2\left[\ln J_{\alpha}(r)\right]^{\prime}+r\left[\ln J_{\alpha}(r)\right]^{\prime \prime}$. Since $J_{\alpha}(r)$ is strictly increasing in $(-\infty, \infty)$ and strictly logarithmically concave in $(-\alpha / 2, \infty)$, it follows that $\left[\ln J_{\alpha}(r)\right]^{\prime}>0$ and $\left[\ln J_{\alpha}(r)\right]^{\prime \prime}<0$ in $(-\alpha / 2, \infty)$. Therefore, $\left[r \ln J_{\alpha}(r)\right]^{\prime \prime}>0$ and $r \ln J_{\alpha}(r)$ is strictly convex in $(-\alpha / 2,0)$.

Remark. If $\alpha=1$, then $r \ln J(r)$ is strictly convex in $(-1 / 2,0)$. This partially answers the question raised by Alzer in [3].

\section{Open Problems}

Finally, we pose the following

Open Problem 1. The generalized one-parameter mean values $J_{\alpha}(r)$ defined by (10) are strictly concave in $(-\alpha / 2, \infty)$.

Open Problem 2. The function $\mathcal{J}_{\alpha}(t)=J_{\alpha}(t) J_{\alpha}(-t)$ is strictly logarithmically convex for $t \notin\left[-\frac{\alpha}{2}, \frac{\alpha}{2}\right]$ and strictly concave and strictly logarithmically concave for $t \in(-\alpha / 2, \alpha / 2)$.

Open Problem 3. Discuss the monotonic and (logarithmically) convex properties of the function $J_{\alpha}(r)+J_{\alpha}(-r)$.

\section{ACKNOWLEDGMENT}

The authors would like to express their many thanks to Professor H. M. Srivastava and Dr. A. Witkowski for their valuable comments on this paper. 


\title{
REFERENCES
}

1. H. Alzer, On Stolarsky's mean value family, Internat. J. Math. Ed. Sci. Tech., 20(1) (1987), 186-189.

2. H. Alzer, Uer eine einparametrige familie von Mitlewerten, Bayer. Akad. Wiss. Math. Natur. Kl. Sitzungsber., 1987 (1988), 23-29. (in German).

3. H. Alzer, Uer eine einparametrige familie von Mitlewerten II, Bayer. Akad. Wiss. Math. Natur. Kl. Sitzungsber., 1988 (1989), 23-29. (in German).

4. W.-S. Cheung and F. Qi, Logarithmic convexity of the one-parameter mean values, RGMIA Res. Rep. Coll., 7(2) (2004), no. 2, Art. 15, 331-342.

5. F. Qi, Logarithmic convexity of extended mean values, Proc. Amer. Math. Soc., 130(6) (2002), n 1787-1796; RGMIA Res. Rep. Coll., 2(5) (1999), Art. 5, 643-652.

6. F. Qi, A note on Schur-convexity of extended mean values, Rocky Mountain J. Math., 35(5) (2005), 1787-1793; RGMIA Res. Rep. Coll., 4(4) (2001), Art. 4, 529-533.

7. F. Qi, The extended mean values: definition, properties, monotonicities, comparison, convexities, generalizations, and applications, Cubo Mat. Ed., 5(3) (2003), 63-90; RGMIA Res. Rep. Coll., 5(1) (2002), Art. 5, 57-80.

8. F. Qi, J. Sándor, S. S. Dragomir, and A. Sofo, Notes on the Schur-convexity of the extended mean values, Taiwanese J. Math., 9(3) (2005), 411-420; RGMIA Res. Rep. Coll., 5(1) (2002), Art. 3, 19-27.

\author{
Wing-Sum Cheung \\ Department of Mathematics, \\ The University of Hong Kong, \\ Pokfulam Road, Hong Kong, \\ People's Republic of China \\ E-mail: wscheung@hkucc.hku.hk \\ Feng Qi \\ Research Institute of Mathematical Inequality Theory, \\ Henan Polytechnic University, \\ Jiaozuo City, Henan Province 454010, \\ People's Republic of China \\ E-mail: qifeng@hpu.edu.cn
}

